\title{
Open Chain Isokinetic Assessment and Exercise of the Knee
}

\author{
By: David H. Perrin
}

Perrin, D.H. (1994). Open chain isokinetic assessment and exercise of the knee. Journal of Sport Rehabilitation, 3:245-254.

Made available courtesy of Human Kinetics:

http://www.humankinetics.com/JSR/journalAbout.cfm

\section{***Note: Figures may be missing from this format of the document}

\begin{abstract}
:
This paper reviews the concepts associated with isokinetic open chain assessment and exereise of the quadriceps and hamstring muscle groups. Following a review of the isokinetic concept of exercise, the paper addresses principles of assessment and exercise of the knee, including the importance of musculoskeletal and cardiovascular screening, warm-up, body position, stabilization, and joint alignment. Gravity correction, test and exercise velocity, and duration of exercise are also addressed. Interpretation of an isokinetic evaluation of the knee is also addressed within the context of force - velocity relationships, peak torque relative to body weight, and bilateral and reciprocal muscle group relationships. Joint range of motion and test velocity are also discussed with respect to patellofemoral and tibiofemoral joint forces. Finally, recommended protocols for isokinetic assessment and exercise of the quadriceps and hamstring muscle groups are presented.
\end{abstract}

\section{Article:}

Isokinetic resistance is a dynamic, fixed-velocity, and accommodating- resistance form of exercise. The primary advantage of isokinetic resistance is that a muscle group may be exercised to its maximum potential throughout the knee joint's entire range of available motion. Isokinetic exercise may be used to quantify the quadriceps and hamstring muscle groups' abilities to generate torque or force and is also useful as an exercise modality in the restoration of either muscle group's preinjury level of strength. A comprehensive review of the theory and clinical application of isokinetic exercise and assessment for all major joints of the body may be found elsewhere (16).

The knee joint is a modified hinge joint formed by the articulation of the distal femur and proximal tibia. The joint is capable of flexion and extension, and it is modified in the sense that internal rotation and external rotation of the tibia occur in combination with flexion and extension, respectively. Twelve muscles croSS the knee joint with the principle extensors being the quadriceps femoris muscles, consisting of the rectus femoris, and the vasti medialis, intermedius, and lateralis muscles. The primary flexors of the knee include the hamstring muscle group, consisting of the biceps femoris, semitendinosus, and 
Table 1 Système International d'Unités (SI)

\begin{tabular}{lll}
\hline Unit & & Conversion \\
\hline Torque & $(\mathrm{ft} \cdot \mathrm{lb}) \times 1.36=(\mathrm{N} \cdot \mathrm{m})$ & $(\mathrm{N} \cdot \mathrm{m}) \times 0.74=(\mathrm{ft} \cdot \mathrm{lb})$ \\
Work & $(\mathrm{ft} \cdot \mathrm{lb}) \times 0.74=(\mathrm{J})$ & $(\mathrm{J}) \times 1.36(\mathrm{ft} \cdot \mathrm{lb})$ \\
Power & $(\mathrm{ft} \cdot \mathrm{lb} / \mathrm{s}) \times 1.36=(\mathrm{W})$ & $(\mathrm{W}) \times 0.74=(\mathrm{ft} \cdot \mathrm{lb} / \mathrm{s})$ \\
\hline
\end{tabular}

Note. $\mathrm{ft} \cdot \mathrm{lb}=$ foot pound; $\mathrm{N} \cdot \mathrm{m}=$ newton meter; $\mathrm{J}=$ joule; $\mathrm{ft} \cdot \mathrm{lb} / \mathrm{s}=$ foot pound $/ \mathrm{sec}$ ond; $\mathrm{W}=$ watt.

semimembranosus muscles. The role of the gastrocnemius muscle in assisting with knee flexion should not be forgotten during rehabilitation of the joint.

A muscle has only the capacity to generate tension or to relax. If the force produced by a muscle is measured about a joint's axis of rotation, the moment of force is known as torque. Torque may be measured as a peak value from the highest point of a given torque curve, or it may be expressed as an average value from each point along the entire curve. If the force and distance of a given muscle contraction are known, the tension produced by a muscle is expressed as work. If the quantity of time required to produce work is known, the ability of the muscle to generate power may be determined. Some clinicians believe that assessment of torque at slow isokinetic test velocities reflects "strength," while the torque produced at high test velocities represents "power." However, torque, power, and work may be assessed at slow, intermediate, or fast isokinetic test velocities. The Système International d'Unités (SI) is the preferred method of reporting isokinetic values and is presented (with conversions) in Table 1.

\section{Interpretation of the Torque Curve}

From the isokinetic torque curve, the clinician is able to determine peak or average torque, work, and power. Computer interfacing with isokinetic dynamometers enables rapid and reliable quantification of these values (17). Since isokinetic resistance accommodates to pain, artifacts may be seen in a torque curve obtained from a patient experiencing pain in the knee joint during assessment. Moreover, some clinicians believe that pathology within a muscle - tendon unit or bony articulation manifests itself with characteristic artifacts within an isokinetic torque curve. For example, some claim the ability to diagnose injury to the anterior cruciate ligament by the appearance of a bimodal torque curve. However, little scientific evidence exists to validate this dubious practice. As such, clinicians are advised to confine their interpretation of the torque curve to a muscle's ability to produce torque, work, and power.

\section{Principles of Isokinetic Assessment and Exercise of the Knee}

The mechanical reliability of several isokinetic dynamometers in the measurement of torque, velocity, and angular position has been reported in the literature $(1,5,22)$. The isokinetic assessment of humans is somewhat more complex, although with adherence to several sound principles, excellent reliability may be obtained during testing of the knee (17). The following principles should be considered prior to isokinetic exercise or assessment of the quadriceps and hamstring muscle groups. 


\section{Musculoskeletal and Cardiovascular Screening}

All patients should be screened for a healthy knee joint before undergoing isokinetic exercise or assessment. For example, both the quadriceps and hamstring muscle groups should be free from spasm or muscle contracture. In addition, the knee joint should be free from swelling, inflammation, and pain. Assessment of a patient experiencing pain in the knee joint may very well reveal false-positive defIcits in strength of either the quadriceps or hamstring muscle groups.

The demands of isokinetic assessment on the cardiovascular system should also be considered. For example, an elderly patient, a severely obese patient, or a patient significantly deconditioned as a result of alienation of the lower extremity should be carefully screened before undergoing isokinetic exercise or assessment. Certain medications may also preclude some patients from undergoing isokinetic exercise or assessment. For example, one report in the literature described a massive hematoma that resulted in the leg of a patient who was receiving the anticoagulant coumadin (20). This case certainly illustrates the importance of taking a careful history before subjecting older patients to the stresses of isokinetic resistance.

Once both the musculoskeletal and cardiovascular systems have been cleared, the patient is ready for the isokinetic form of resistance.

\section{Patient Education, Familiarization, and Warm-Up}

Since isokinetic resistance is a novel sensation for all people, each patient should receive a verbal orientation to this form of exercise therapy. The need for this orientation has become even more necessary with the creation of "active" isokinetic dynamometry, which enables exercise and assessment through both concentric and eccentric modes of contraction. In the case of concentric contraction, patients should be instructed to straighten or bend the knee as hard as they can. For the eccentric mode of resistance, they should be informed to resist the dynamometer as it attempts to push or pull the knee.

Warm-up should also precede isokinetic assessment or exercise of the knee. Static stretching of both the quadriceps and hamstring muscle groups is indicated, as well as general body warm-up in the form of submaximal stationary cycling. Patients should also be provided both submaximal and maximal repetitions for warm-up purposes. A sequence of three submaximal and three maximal warm-up repetitions prior to assessment at each isokinetic test velocity has been associated with reliable isokinetic assessment (17).

\section{Body Position, Stabilization, and Joint Alignment}

For optimal isolation of the quadriceps and hamstring muscle groups, patients should be positioned and stabilized with straps on the dynamometer at the chest, waist, and thigh. The arms should be crossed over the chest to minimize any contribution from accessory muscle groups that might result from pushing and pulling with the hands. Finally, the axes of rotation of the dynamometer and the knee joint should be aligned as closely as possible.

Most clinicians assess and exercise the quadriceps and hamstring muscle groups while patients are seated, and most normative data in the literature were obtained from this position. However, the hamstring muscles are capable of producing higher levels of both concentric and eccentric 
torque from the prone position than the supine position, and either of these positions more closely simulates the length-tension relationship of both the quadriceps and hamstring muscle groups during activities such as walking and running (25). Clinicians are advised to consider these alternative positions during both exercise and assessment of the quadriceps and hamstring muscle groups.

\section{Gravity Correction}

Gravitational force tends to enhance the strength of muscles assisted by gravity and to detract from muscles opposed by gravity. In the case of the thigh musculature, quadriceps force may be underpredicted by 4 to $43 \%$ and hamstring force overpredicted by 15 to $519 \%(14,24)$. Thus the importance of gravity correction in obtaining valid strength measures of these muscle groups has been established $(2,18,21,24)$. Failure to correct for the effects of gravity also confounds determination of the hamstring/quadriceps reciprocal muscle group ratio. Since gravity correction tends to reduce hamstring torque and increase quadriceps torque, determination of the ratio from uncorrected values will in fact inflate the ratio $(6,7)$.

\section{Velocity Spectrum Exercise}

Rehabilitation protocols often include exercise over a range of angular velocities, which has been termed "velocity spectrum" exercise. While many clinicians empirically report positive results with such a regimen, the physiological rationale for this principle is not well established in the scientific literature. Two possible explanations exist and appear to be related either to differential muscle fibers or to recruitment patterns within the quadriceps and hamstring muscle groups. The muscle fiber theory would suggest that since slow twitch fibers are specialized for use at relatively slow velocities and fast twitch fibers are specialized for high velocities, the fiber types are selectively recruited during slow and fast isokinetic exercise, respectively. On the other hand, since isokinetic exercise is a maximal form of accommodating resistance requiring high levels of muscular tension, both slow and fast twitch fibers would be expected to be recruited at both slow and fast speeds of movement. Further research is needed to clarify this apparent contradiction in theories.

\section{Duration of Exercise}

Duration of isokinetic exercise is of prime importance during rehabilitation of the quadriceps and hamstring muscle groups. For example, a comparison of 6 and $30 \mathrm{~s}$ of isokinetic knee extension exercise reveals substantial increases in muscle lactate only after $30 \mathrm{~s}$ (3). The duration of isokinetic exercise should also be based on the element of time rather than number of repetitions. For example, 30 repetitions at $90^{\circ} / \mathrm{s}$ through a $90^{\circ}$ arc of motion takes $30 \mathrm{~s}$, while 30 repetitions at $180^{\circ} / \mathrm{s}$ through the same range of motion takes only $15 \mathrm{~s}$.

\section{Recommended Test and Exercise Protocols}

A wide range of test and exercise protocols exists among clinicians who incorporate the isokinetic concept of exercise in rehabilitation of the knee. In general, isokinetic protocols are either continuous or interrupted. Interrupted protocols allow a pause of several seconds between contractions, while continuous protocols do not permit a rest interval between subsequent contractions. For either continuous or interrupted protocols, several combinations of agonist/agonist or agonist/ antagonist contractions are available such as concentric/concentric, eccentric/ eccentric, concentric/eccentric, or eccentric/concentric. 
Recent research has examined the effect of test protocol and isokinetic device on quadriceps torque and found that both protocols are reproducible over time. However, greater torque was generated by the quadriceps with an interrupted protocol regardless of exercise device (8). In all probability, the use of consistent protocols supersedes the exact nature of the protocol used, primarily for assessment, but for exercise as well. The following lists provide guidelines in the design of isokinetic test and exercise protocols for the quadriceps and hamstring muscle groups.

\section{Recommended Protocol for Concentric and Eccentric}

\section{Isokinetic Assessment of the Quadriceps and Hamstring Muscle Groups}

- Musculoskeletal screening of quadriceps and hamstring muscle groups and knee joint

- General body stretching and warm-up

- Patient setup in seated, prone, or supine position

- Alignment of joint and dynamometer axes of rotation

- Verbal introduction to isokinetic concept of exercise

- Gravity correction procedure

- Warm-up (3 submaximal, 3 maximal) prior to each test velocity

- 30 s rest

- Warm-up and maximal test at slow velocity (4-6 repetitions)

- 30 s rest

- Warm-up and maximal test at fast velocity (4-6 repetitions)

- 30 s rest

- Warm-up and multiple-repetition endurance test

- 60 s rest

- Testing of contralateral extremity

- Recording of test details to ensure replication on retest

- Explanation of results to patient

\section{Recommended Exercise Protocol}

\section{for the Quadriceps and Hamstring Muscle Groups}

- Musculoskeletal screening of quadriceps and hamstring muscle groups and knee joint

- General body stretching and warm-up

- Patient setup with optimal stabilization

- Alignment of joint and dynamometer axes of rotation

- Submaximal warm-up for familiariZation with exercise velocity

- $30 \mathrm{~s}$ concentric and eccentric exercise at slow velocity

- Rest

- Warm-up and 30s concentric and eccentric exercise at intermediate velocity

- Rest

- Warm-up and $30 \mathrm{~s}$ concentric and eccentric exercise at fast velocity

- Rest

- Repeated exercise bouts from seated and prone or supine positions

- General body stretching and cool-down

- 20 min ice application to involved joint or muscle group 
Isokinetic evaluation of the knee may be used to monitor an injured patient's progress through a rehabilitation program and to determine readiness to return to activities of daily living or athletic participation. Interpretation of the isokinetic evaluation necessitates careful analysis of the quadriceps and hamstring muscles' abilities to generate torque, power, and/or work. Torque may be assessed as an average or peak value of concentric or eccentric force.

\section{Force-Velocity Relationships}

The ability of the quadriceps and hamstring muscles to produce concentric torque is greatest at slow isokinetic test velocities and will decrease linearly as test velocity increases. Eccentric peak torque tends to be greater than concentric torque at slow velocities and will not decrease with increases in test velocity. Some differences exist in the eccentric force-velocity curve between intact human muscle and isolated animal muscle. For example, the classic force-velocity curve described by Hill (9) and derived from isolated animal muscle showed decreases in concentric force with increases in velocity, and increases in eccentric force with increases in velocity. However, eccentric force usually exceeds isometric levels in humans but then increases only slightly with increases in velocity.

This difference in the force - velocity curve has been postulated to be related to a neural inhibitory safety mechanism in humans that prevents injury to muscle during eccentric contractions at high velocity (23). The clinical implication is that decreases in concentric torque with an increase in test velocity are a normal finding. In contrast, eccentric torque should be expected to increase slightly above isometric strength levels but will not continue to increase with an increase in test velocity. A decline in eccentric strength with an increase in test velocity should be interpreted as an abnormal finding. An extensive listing of normative data for the quadriceps and hamstring muscle groups for several populations at a variety of isokinetic test velocities may be found elsewhere (16).

\section{Peak Torque Relative to Body Weight}

One special difficulty in comparing normative strength values among patients is the confounding influence of body size. To account for marked differences in body size, strength may be expressed as a percentage of total body weight (26). In fact, the software of some isokinetic dynamometers automatically computes and reports peak torque values of the quadriceps and hamstring muscle groups divided by the patient's body weight. Strength values normalized to body weight for a variety of populations at several isokinetic test velocities may be found elsewhere (16), although caution is advised when interpreting these ratios. For example, values expressed as $\mathrm{ft} \mathrm{lb}$ torque/lb body weight yield very different ratios than when expressed as $\mathrm{N} \mathrm{m}$ torque $/ \mathrm{kg}$ body weight. These differences are related to the unit of measurement rather than to actual differences in the peak torque to body weight ratio.

\section{Bilateral and Reciprocal Muscle Group Comparisons}

Comparison of the injured extremity's strength with that of the uninjured extremity is commonly used as the standard for return of the injured side to a normal state following rehabilitation (19). That is, the injured side quadriceps muscle group is compared with the uninjured side quadriceps, and the injured side hamstring muscle group is compared with the uninjured side hamstrings. For most populations, values for right and left side quadriceps and hamstring muscle group strength are usually within 5 to $10 \%$ of each other. However, caution is advised when 
making this comparison in athletes involved in asymmetrical activities such as kicking or jumping from one leg.

To produce extension of the knee, the quadriceps muscle group acts as the agonist and the hamstring muscle group acts as the antagonist. The opposite occurs during flexion of the knee; that is, the hamstring muscle group is the agonist and the quadriceps muscle group is the antagonist. The relationship of the hamstring to the quadriceps is known as the reciprocal muscle group ratio. Although the quadriceps muscles are naturally stronger than the hamstrings, it has been assumed by many clinicians (although not scientifically proven) that gross imbalances between these muscle groups predispose one to injury. Thus the hamstring/quadriceps reciprocal muscle group relationship has received a considerable amount of attention during rehabilitation of the knee. The reciprocal muscle group ratio is determined quite easily by dividing the strength of the hamstrings by the strength of the quadriceps. However, determination of a normal ratio may be confounded by several factors, including test velocity and presence or absence of a gravity correction procedure. For example, a value of .60 is quite normal for the hamstring/quadriceps ratio at a slow isokinetic test velocity in some populations, but this value typically increases significantly as test velocity increases. Use of gravity correction helps to adjust for this error when determining the ratio throughout a velocity spectrum of testing. A comprehensive list of normal hamstring/quadriceps reciprocal muscle group ratios for a variety of populations across several isokinetic test velocities is available elsewhere (16).

\section{Special Rehabilitation Considerations}

The goal of rehabilitation of the knee is to return the quadriceps and hamstring muscle groups to normal preinjury levels of strength, with special reference to the bilateral and reciprocal muscle group relationships. However, the complexity of the knee joint may create special challenges to the clinician during the rehabilitation of a variety of industrial or athletic injuries. Patellofemoral and tibiofemoral joint forces must be considered, and the role of weight-bearing activities in a comprehensive rehabilitation program deserves attention.

\section{Patellofemoral Joint Forces}

A healthy patellofemoral articulation is essential to the normal biomechanics of the knee joint. Patellofemoral joint pain not only can confound accurate assessment of the quadriceps and hamstring muscle groups but can also impede the return of these muscle groups to preinjury levels of strength. Many clinicians have observed that isokinetic exercise at slow velocities through a full range of knee motion tends to exacerbate the symptoms of patellofemoral pain. This clinical observation has at least in part been confirmed through scientific investigation. Kaufman et al. (11) used a mathematical model to calculate the patellofemoral compressive forces during full-range isokinetic exercise of the knee at 60 and $180^{\circ} / \mathrm{s}$. In general, compressive forces were found to be low at knee flexion angles less than $20^{\circ}$ and were highest at 70 to $75^{\circ}$ of knee flexion, approaching 5.1 times body weight during testing at $60 \%$. These compressive forces were only slightly lower (4.9 times body weight) at $180^{\circ}$ s.

The obvious clinical implication of these findings is that when patellofemoral joint pain is present, full-range isokinetic exercise should be avoided, although limited-range exercises may be indicated in the last $20^{\circ}$ of knee extension. The findings of Kaufman et al. (11) would suggest that the problems of isokinetic exercise in the presence of patellofemoral joint pain are more 
related to range of knee motion than to exercise velocity. This fact would seem to contradict the long-standing clinical impression that joint compressive forces are significantly reduced with increases in joint angular velocity.

\section{Tibiofemoral Joint Forces}

Isokinetic exercise of the quadriceps muscle group with the resistance pad placed at the distal tibia has been shown to create considerable tibiofemoral shear forces. This anterior force acts primarily in the last $40^{\circ}$ of knee extension and is approximately 0.2 to 0.3 times body weight (11). The anterior cruciate ligament is responsible for checking anterior displacement of the tibia on the femur. Thus caution is advised when providing isokinetic resistance during knee extension in patients experiencing injury or reconstruction of the anterior cruciate ligament.

The magnitude of the anterior shear force may be significantly reduced by moving the resistance pad closer to the proximal end of the tibia (15). The pad should be placed proximally during the early phases of isokinetic exercise during rehabilitation of the anterior cruciate ligament injured or reconstructed knee, and should be moved distally along the tibia in gradual increments. A dual-pad resistance device is also commercially available to reduce this anterior shear force (10). The proximal placement or dual-pad device should be used during knee extension and not during knee flexion, as the anterior shear may actually be increased with proximal placement during knee flexion exercises.

\section{Closed Chain Exercises}

Isokinetic resistance is very effective in returning the strength of the quadriceps and hamstring muscle groups to preinjury levels. However, a comprehensive rehabilitation program should also include strengthening and functional activities from a weight-bearing position, which has been termed "closed chain" exercise. An isokinetic dynamometer may be used as a leg press device (4, 13), or simple isotonic activities such as squats may be incorporated into the rehabilitation program. Clinicians are also advised to incorporate a variety of weight-bearing functional activities into the program, which may provide exercise and allow the clinician to assess progress through rehabilitation (12).

\section{Summary}

Isokinetic resistance is a dynamic, fixed-velocity, and accommodating-resistance form of exercise that may be used for assessment and exercise of the quadriceps and hamstring muscle groups. Reliable assessment necessitates adherence to several principles related to familiarization and warm-up, body position, stabilization, joint alignment, and gravity correction. Interpretation of the isokinetic evaluation includes assessment of peak torque with respect to bilateral and reciprocal muscle group relationships. Sound exercise programs are based on velocity spectrum exercise, adequate duration of exercise, and attention to the patellofemoral and tibiofemoral joint forces associated with isokinetic resistance.

\section{References}

1. Bemben, M.G., K.J. Grump, and B.H. Massey. Assessment of technical accuracy of the Cybex II isokinetic dynamometer and analog recording system. J. Orthop. Sports Phys. Ther. 10:12-17, 1988. 
2. Bohannon, R.W., and M.B. Smith. Intrasession reliability of angle specific knee extension torque measurements with gravity corrections. J. Orthop. Sports Phys. Ther. 11:155-157, 1989.

3. Costill, D.L., E.F. Coyle, W.F. Fink, G.R. Lesmes, and F.A. Witzman. Adaptations in skeletal muscle following strength training. J. Appl. Physiol. 46:96-99. 1979.

4. Engle, B. Clinical use of an isokinetic leg press. J. Orthop. Sports Phys. Ther. 4:148149, 1983.

5. Farrell, M., and J.G. Richards. Analysis of the reliability and validity of the kinetic communicator exercise device. Med. Sci. Sports Exerc. 18:44-49, 1986.

6. Figoni, S.F., C.B. Christ, and B.H. Massey. Effects of speed, hip and knee angle, and gravity on hamstring to quadriceps torque ratios. J. Orthop. Sports Phys. Ther. 9:287-291, 1988.

7. Fillyaw, M., T. Bevins, and L. Fernandez. Importance of correcting isokinetic peak torque for the effect of gravity when calculating knee flexor to extensor musele ratios. Phys. Ther. 66:23-29, 1986.

8. Heinrichs, K.I., D.H. Perrin, A. Wellman, J.H. Gieck, and D.W. Ball. Effect of protocol and assessment device on isokinetic peak torque of the quadriceps muscle group. In review.

9. Hill, A.V. The heat of shortening and the dynamic constants of muscle. Proc. R. Soc. Lond. (Biol.) 126:136-195, 1938.

10. Johnson, D. Controlling anterior shear during isokinetic knee extension exercise. J. Orthop. Sports Phys. Ther. 4:23-31, 1982.

11. Kaufman, K.R., K.N. An, W.J. Litchy, B.F. Money, and E.Y.S. Chao. Dynamic joint forces during knee extension exercise. Am. J. Sports Med. 19:305-316, 1991.

12. Lephart, S.M., D.H. Perrin, K. Minger, and F.H. Fu. Sports specific functional tests for the anterior cruciate ligament insufficient athlete. Athl. Training 26:44-50, 1991.

13. Levine, D., A. Klein, and M. Morrissey. Reliability of isokinetic coneentric closed kinematie ehain testing of the hip and knee extensors. Isokin. Exerc. Sci. 1:146-152, 1991.

14. Nelson, S.G., and P.W. Duncan. Correetion of isokinetic and isometric torque recordings for the effects of gravity. Phys. Ther. 63:674-676, 1983.

15. Nisell, R., M.O. Erieson, G. Nemeth, and J. Ekholm. Tibiofemoral joint forces during isokinetic knee extension. Am. J. Sports Med. 17:49-54, 1989.

16. Perrin, D.H. Isokinetic Exercise and Assessment. Champaign, IL: Human Kinetics, 1993.

17. Perrin, D.H. Reliability of isokinetic measures. Athl. Training 10:319-321, 1986.

18. Perrin, D.H., E.M. Haskvitz, and A. Weltman. Effect of gravity correction on isokinetic average force of the quadriceps and hamstring muscle groups in women runners. Isokin. Exerc. Sci. 1:99-102, 1991.

19. Perrin, D.H., R.J. Robertson, and R.L. Ray. Bilateral isokinetic peak torque, torque acceleration energy, power, and work relationships in athletes and nonathletes. J. Orthop. Sports Phys. Ther. 9:184-189, 1987.

20. Richter, K.J. Subcutaneous hemorrhage in a patient on coumadin: An isokinetic exercise complication. J. Sport Rehabil. 1:264-266, 1992.

21. Rothstein, J.M., R.L. Lamb, and T.P. Mayhew. Clinical uses of isokinetic measurements. Phys. Ther. 67:1840-1844, 1987.

22. Taylor, N.A.S., R.H. Sanders, E.I. Howick, and S.N. Stanley. Static and dynamic assessment of the biodex dynamometer. Eur. J. Appl. Physiol. 62:180-188, 1991.

23. Westing, S.H., A.G. Cresswell, and A. Thorstensson. Muscle activation during maximal voluntary eccentric and coneentric knee extension. Eur. J. Appl. Physiol. 62:104108, 1991. 
24. Winter, D.A., R.P. Wells, and G.W. On. Errors in the use of isokinetic dynamometers. Eur. J. Appl. Physiol. 46:397-408, 1981.

25. Worrell, T.W., C.R. Denegar, S.L. Armstrong, and D.H. Perrin. Effect of body position on hamstring muscle group average torque. J. Orthop. Sports Phys. Ther. 11:449451, 1990.

26. Worrell, T.W., D.H. Perrin, B.M. Gansneder, and J.H. Gieck. Comparison of isokinetic strength and flexibility measures between hamstring injured and non-injured athletes. $J$. Orthop. Sports Phys. Ther. 13:118-125, 1991. 